\title{
Extraction of cardiac and respiration signals in electrical impedance tomography based on independent component analysis
}

\author{
T. Rahman ${ }^{1,4}$, M. M. Hasan ${ }^{2}$, A. Farooq ${ }^{1}$ and M. Z. Uddin ${ }^{3}$ \\ 1. Department of Biomedical Engineering, Kyung Hee University, South Korea \\ 2. School of Engineering \& Information Technology, University of New South Wales, Australia \\ 3. Electronic and Electrical Engineering, Inha University, South Korea \\ 4. E-mail any correspondence to: tasneem@khu.ac.kr
}

\begin{abstract}
Electrical Impedance Tomography (EIT) has successive wide range in impedance imaging, but still it is difficult to extract cardiac-related conductivity changes and respiratory-related conductivity changes in spontaneous breathing subjects. Quite a few methods are attempted to extract these two signals such as electrocardiogram gated averaging, frequency domain filtering and principal component analysis. However, such methods are not able to take apart these components properly or put some effort in real time imaging and have their own limitations. The purpose of this paper is to introduce a new method in the EIT clinical application field, Independent Component Analysis (ICA) to extract cardiac and respiratory related signals in electrical impedance tomography. Independent component analysis has been introduced to use in electrical impedance tomography but this is the first attempt ever to implement this method to separate these two signals and image those independent conductivity distribution of respiration and cardiac changes independently. Data has been collected from a spontaneous breathing subject. Filtration technique has been used to remove random noise and multi level spatial ICA has been applied to obtain independent component signals which has been later used in reconstruction algorithm for imaging.
\end{abstract}

Keywords: Biomedical Engineering, Electrical Impedance Tomography, Independent Component Analysis, Image Reconstruction, Cardiac Signal, Respiration Signal.

\section{Introduction}

Electrical impedance tomography (EIT) is an imaging technique to image the impedance changes of the human body. It produces cross-sectional images of a conductivity distribution inside the human body using measured boundary voltage data sets which typically use a single ring external electrode. EIT has been widely used for functional imaging associated with the different physiological changes such as cardiac and respiratory changes. The first working device of EIT was presented by Brown et al and Seagar et al [1,2] and afterwards it has been developed significantly and used for some clinical applications. Yet it is too far to use EIT in daily clinical use. Beside other applications, the use of EIT for monitoring regional ventilation changes has been done in several research studies [3-6]. In Fig. 1 we have shown the basic EIT arrangement. Sixteen electrodes are attached in equidistance. In interrogated body section current (I) is injected in one pair and voltage (V) is sensed by other pair of electrodes. Neighboring current pattern is used for every injected pair of current and rests of the electrodes are used to sense the voltage.

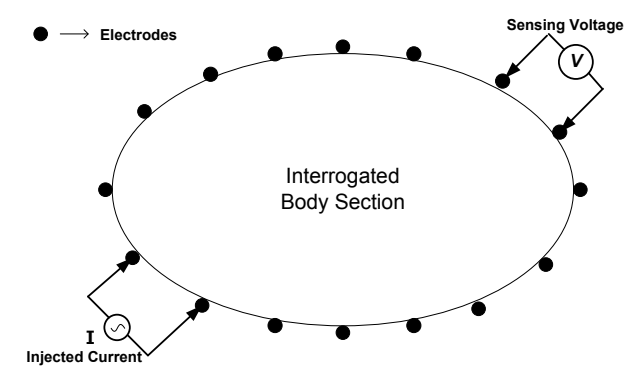

Fig. 1: Basic EIT arrangement.

Electrical impedance tomography (EIT) is a non-invasive, inexpensive and portable imaging modality which has a promising future to be used for bedside monitoring $[7,8]$. Numerous attempts have been taken earlier to extract cardiac and respiration signals properly. However those methods have their own limitations and are not satisfactory to obtain proper independent signal of cardiac and respiration signal. Consequently Independent Component Analysis (ICA) is a way to resolve signals into independent components based on statistical characteristics of the signals [9]. This method has been successfully pertained in EIT before. The EIT recordings are the result of linear combinations of the source signals. Using ICA the signal subspace will then be decomposed into statistically independent components [9]. At the present it is high time to apply this method in some extended clinical applications of EIT.

To reconstruct images from EIT datasets it is necessary to get all the individual conductivity distribution of the source signal. ICA is a method for factoring probability densities of measured signals into a set of densities that are as statistically independent as possible under the assumptions of a linear model. The EIT datasets are the result of linear combinations of the source signals. Using ICA the signal subspace we will decompose the linearly combined source signals into statistically independent components. Independent component analysis (ICA) is one of the most widely used Blind Source Separation (BSS) 
techniques for revealing hidden factors that underlie sets of random signals. It extracts individual signals from mixtures shown in Fig. 2.

In this paper we want to propose a multi level spatial ICA based method that has been applied to acquire separate independent cardiac and respiration signal properly. This is the first ever attempt to extend the EIT based applications in the bed side monitoring. Firstly ICA gives the template function of most prominent respiration signal and again in the second level approximation ICA is been used to get another template function of cardiac signal from the time domain filtered signal. Then we analyze the template signals by using Fast Fourier Transform. Both of these template functions are later used in a reconstruction algorithm to get the impedance image. Also the computational time of multi-level spatial ICA is found reasonable to use this technique in real time system for bed side monitoring.

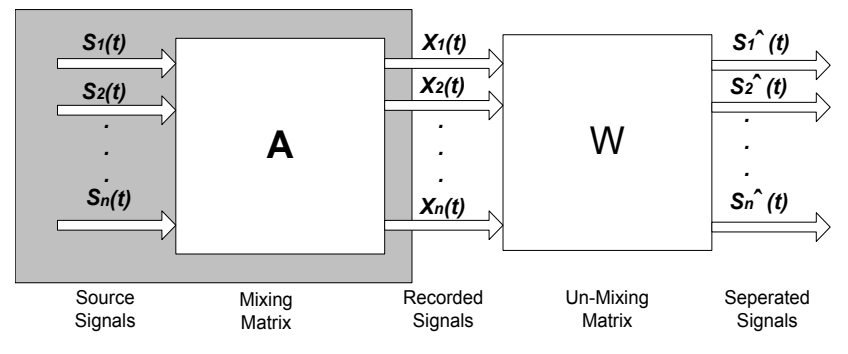

Fig. 2: Independent Component Analysis block diagram where blind source $s^{\wedge}(t)$ is estimated from a mixing matrix $A . s(t)$ are the sources. $x(t)$ are the recordings, $s^{\wedge}(t)$ are the estimated sources. Also $A$ is mixing matrix and $W$ is un-mixing matrix.

\section{Previous Relevant Research}

To study the previous relevant research, we organize our background study in three different existing approaches; ECG Gated Acquisition, Frequency Domain Filtering and recent PCA Based Methods.

\section{ECG Gated Acquisition}

ECG gated method is the first approach of separation of cardiac and respiration changes in electrical impedance tomography by McArdle et al [10]. It is based on two significant criteria. First, the impulse component of perfusion signal in the lung is in synchronization with cardiac changes and therefore the ECG. Second, the ventilation changes in impedance occur at a much lower rate compared to the ECG. Thus, by recording the changes of impedance during the cardiac cycle, triggered by a separated ECG module, the lung perfusion signal can be recorded. Furthermore, it has been shown in various studies that averaging the results over more than 100 cardiac cycles can reduce the interference of respiration. Averaging the measurements over 100 cardiac cycles is used in [11] and [12], where some cases averaging has been done for over 200 cardiac cycles [13]. ECG-gated acquisition demands higher frame rates from the EIT instrument to capture the changes within a cardiac cycle. New generation EIT systems have overcome these demerits. The averaging over a large number of cardiac cycles requires long duration of data acquisition and therefore may not be suitable for continuous bedside or real-time monitoring. Furthermore, as Deibele et al [14] pointed out, this averaging over a large data set could result in the fact that 'sudden changes or irregular anomalies cannot be detected'. In addition, with this method of acquisition, the ventilation component of the signal is lost, which is certainly not the optimal solution.

\section{Frequency Domain Filtering}

These methods are based on the fact that the heart rate and the respiratory rate are very well separated in frequency. The fast Fourier transform (FFT) of the global signal can provide a good approximation of the required cut-off frequency for filtering as shown in Fig. 3 showed by Grant et al [15]. Accordingly, FIR or IIR filters can be implemented as a high-pass filter to extract the cardiacrelated component from the mixed signal. Similarly, the respiratory component of the signal can be extracted using a low-pass filter, possibly with the same cut-off frequency. This filtration process can be carried out on the voltage signals or the reconstructed images [16].

In spontaneously breathing subjects, the respiratory impedance changes are rarely sinusoidal, leading to frequency components across a wider range of spectrum than anticipated. Frerichs et al [17] and Grant et al [15] used frequency domain filtering to first separate components in the heart-rate domain and the respiratory rate domain, followed by linear regression fit to identify the regions of interest (ROIs). Whilst [17] used mechanically ventilated subjects to ensure no overlapping in the frequency spectrums of the ventilation and perfusion components of the acquired signal, [15] successfully separated the two components in spontaneously breathing subjects by combining the phase component of the signals with the algorithm suggested by [17].

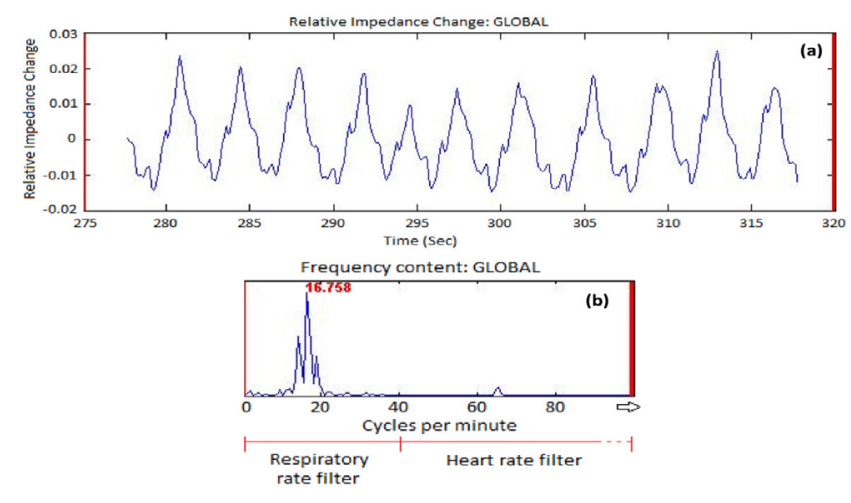

Fig. 3: (a) The original time course of impedance change of a subject during spontaneous breathing with no filtering applied. (b) The Fast Fourier Transform (FFT) power spectrum of this signal showing the frequency characteristics [15]. 


\section{PCA Based Method}

A Principal Component Analysis (PCA) - based method for separating the respiratory and the perfusion-related signal was suggested in [14]. Their method successfully separated the two signals, allowing continuous beat-to-beat updates after a $20 \mathrm{~s}$ period of initiation. Time domain filtering with template functions was used to initially separate the cardiac and the ventilation signal. The results were then combined to obtain the best fit with the original signal, computed by performing least mean square (LMS) between the approximation and the actual signal.

In order to achieve proper separation of the respiratory and cardiac-related signals, the template functions for time domain filtering were identified by performing the PCA on the original data matrix. In [18] each of the aforementioned techniques compares the results achieved by each of these approaches in their experiment. Their results showed that the PCA and the frequency domain filtering methods produced highly correlated results. On the other hand, the correlation between these two methods with the ECGgating alternative was reported considerably less. However, the correlations between each of these methods and a known standard method for perfusion imaging were not computed, rendering the results from their study inconclusive regarding as to which method is more reliable.

\section{Proposed Method Using ICA}

Impedance change of cardiac signal mixes with the impedance change of respiration signal and impedance change of blood flow. The EIT signal gets demodulated with the respiration signal of the human body and the cardiac signal gets demodulated too. As a result the output demodulated signal remains as the mixture of the cardiac and respiration signal together shown in Fig. 4. Based on our proposed method we are towards to put a novel impact in this inconveniency where we are to get the independent cardiac and respiration signal simultaneously. In the following sections our proposed method is described in detail.

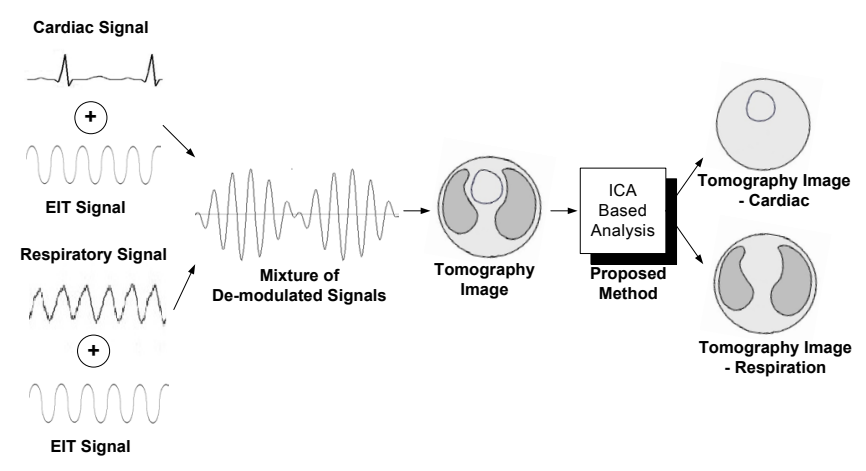

Fig. 4. Separation of Cardiac and Respiration signal

In Fig. 5, we have shown the block diagram of our proposed method steps. Firstly ICA gives the template function of the most prominent respiration signal and again in the second level approximation ICA has been used to get another template function of cardiac signal from the time domain filtered signal. Then we analyze the template signals by using Fast Fourier Transform. Both of these template functions are later used in reconstruction algorithm to get the impedance image.

\section{ICA Algorithm in EIT}

In electrical impedance tomography, we preprocess the acquired data to decompose them into several independent activation maps. The source for each activation map will then be localized independently [9]. To apply ICA algorithm in EIT, we have made some assumptions [9,1921]. At First, the sources must be independent and is valid for our multiple conductivity distribution source of localization problem. The mixture is linear. There is no delay in signal propagation from sources to sensors and finally, the total number of independent signal sources does not exceed the number of electrodes.

\section{Preprocessing}

To apply ICA in EIT we need to do some preprocessing [9]. A useful preprocessing technique in ICA is to whiten the observed signals. Whiten refers such transformation to preliminary sphere the data where the observed vector $x$ is linearly transformed to a new vector $\tilde{x}$ which is white i.e. its components are uncorrelated and the covariance matrix of $x$ equals the identity matrix.

$$
E\left\{\tilde{x} \tilde{x}^{T}\right\}=I
$$

The second step in processing the raw EIT data $\tilde{x}$ is to decompose it into signal and noise subspaces by applying the PCA method in order to reduce the dimensionality of the data and remove some of its noise.

\section{Template Selection Using ICA}

The projection of the data on the signal subspace will be referred to as $V_{\text {source }}$. The signal subspace $V_{\text {source }}$ will then be decomposed into statistically independent components $V_{\text {source }}^{j}$. Then the EIT recordings $V_{\text {source }}$, which are the result of linear combinations of the source signals $S$, can therefore be expressed as:

$$
V_{\text {source }}=M . S
$$

Where $M$ is the so-called mixing matrix. If $U . V_{\text {source }}=S$ or $U=M^{-1}$, then $S$ can be found. Here $U$ is the weighting matrix or unmixing matrix, which 


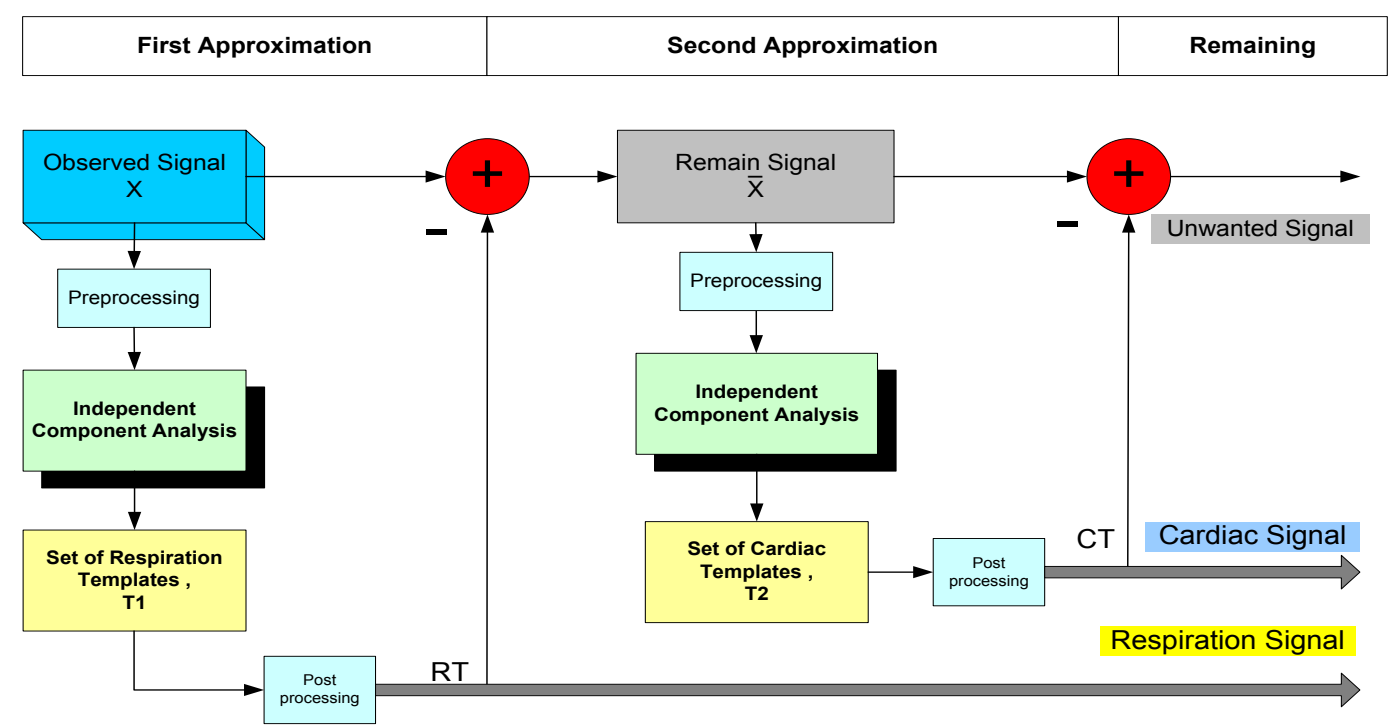

Fig. 5: Proposed method to Separate Cardiac and Respiration signal; After preprocessing ICA is applied on observed signal $x$ to get the respiration template function RT from a set of templates T1 and identically cardiac template function, $\mathrm{CT}$ is obtained from remain signal. Unwanted signals are not taken into consideration.

inverts the mixing process. $\mathrm{M}$ is unknown and the only data we have is the $V_{\text {source }}$ matrix. The ICA process consists of two phases: learning and processing. During the learning phase, the ICA algorithm finds a $U$ which minimizes the mutual information among channels. There exist several different ways to estimate the $U$ matrix. Here the natural gradient vector is used to speed up solution convergence by avoiding the complex computation of matrix inverses [19]. Weighting matrix $U$ can be constructed iteratively by:

$$
U_{i+1}=U_{i}+\alpha_{i} \cdot\left[I+2 G\left(p_{i}\right) \cdot p_{i}^{T}\right] \cdot U_{i}
$$

where the vector $p_{i}$ is defined as,

$$
p_{i}=U_{i} \cdot I_{\text {source }}
$$

and for the nonlinear function $G$ we used,

$$
G\left(p_{i}\right)=\tanh \left(p_{i}\right)
$$

In the equation (3), $\alpha_{i}$ is a learning rate and $I$ is the identity matrix. The learning rate decreases during the iteration and we stop when $\alpha_{i}$ becomes smaller than a predefined tolerance.

The second phase of the ICA algorithm is the actual source separation. Independent components can be computed by applying the $U$ to the signal subspace data.

$$
S=U \cdot V_{\text {source }}
$$

Projection of independent activation maps $S$ back onto the electrodes can be done by:

$$
V_{\text {source }}^{j}=R_{\text {source }} \cdot \sqrt{\lambda} \cdot U^{-1} \cdot S_{k}
$$

where $V_{\text {source }}^{j}$ is the set of sensing voltage due to just the $j$ th source. $\lambda$ and $R_{\text {source }}$ are the signal subspace singular values and singular vectors. For each electrode voltage just the $j$ th source voltage map $V_{\text {source }}^{j}$, we can now reconstruct the different conductivity distribution in different regions using the sensitivity theorem for EIT image.

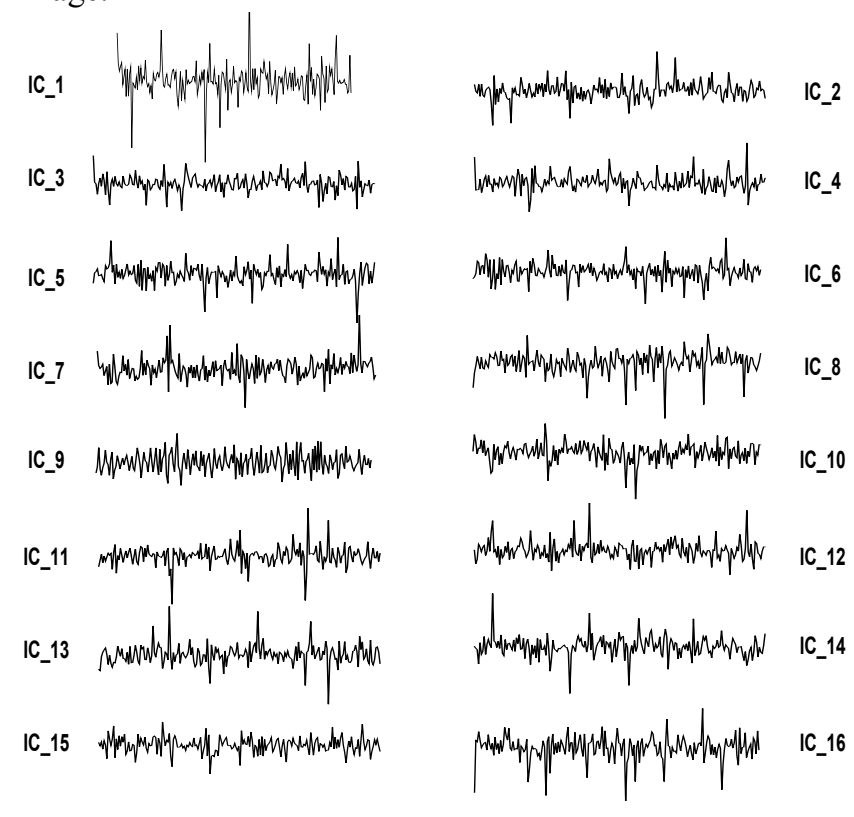

Fig 6. Independent Component signals generated from preprocessed raw EIT data. 


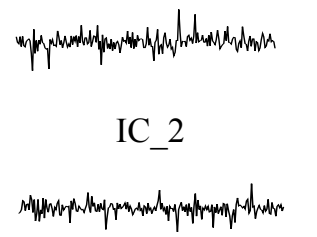

IC_6

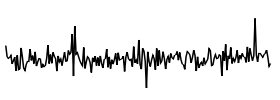 \\ IC_ 7

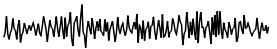

IC_9
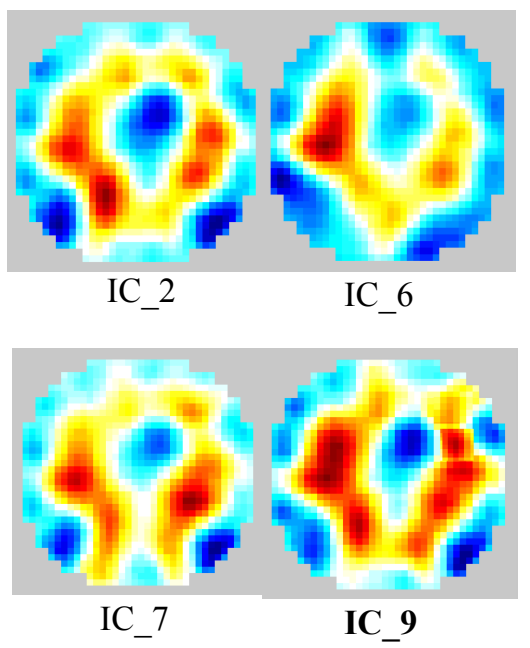

Fig. 7: Selected IC's (left column) and corresponding reconstructed images (right column)

\section{Experimental Results}

Experiment was done on a volunteered human subject. The data were collected from a normal male volunteer, without records of previous lung disease or smoking habits, and presenting a normal radiograph. The volunteer was upright during data collection, quietly breathing, with the electrodes placed at the level of the thoracic plane crossing the fifth intercostal space. Data was recorded using 16 channel EIT device for spontaneous breathing for continuous $40 \mathrm{~s}$.

\section{Experimental Setup}

In order to test the proposed method, data was collected from a human subject. EIT data were acquired using 16 channels KHU Mark2.5 with 16 electrodes. The electrodes were placed circumferentially (equally spaced) around the thorax just below the level of the axilla. An electrical current of $1 \mathrm{~mA}$ was injected at $50 \mathrm{kHz}$ through a pair of neighboring electrodes. Differential voltages were measured between the other non-injecting pair of electrodes with neighboring pattern. Following this initial current injection, the electrical current was then injected sequentially via the next pair of electrodes and repeated until all electrodes had served for current injection. The data for one complete cycle produced a so-called "frame," and they were saved in a raw data file for later processing. The equipment has an SNR of $80 \mathrm{~dB}$.

Considering all current patterns, 256 measurements were obtained. A 2-D finite element mesh with 499 linear triangular elements was built, with shape and size similar to the cross section of the volunteer's thorax. Using the sensitivity matrix algorithm, 1650 measurement vectors were used to generate the transition matrix. Truncated singular valued decomposition (tSVD) algorithm was applied to get the reconstruction images.

\section{Result and Discussion}

In the experimental section, some intermediate results of our algorithm stages is shown and analyzed. For our experiment we first analyzed the input signals of our EIT data. We applied some preprocessing steps like whitening, PCA for denoising and to ensure the input data to be uncorrelated. Then we applied ICA to get the independent signals from our uncorrelated raw data. In Fig. 6 we have shown the sixteen independent components (IC's) generated from ICA algorithm. A typical input signal as can be found in the lung region is depicted. The superposition of a lower frequency ventilation signal and a much smaller higher frequency cardiac signal can be distinguished. The signals are represented as frame numbers (horizontally) along with amplitude (vertically).

All these IC's are representing conductivity distribution up to some extent, which are verified by the Fast Fourier Transformation later on. Firstly, we applied FFT of all the selected IC's generated from ICA algorithm. For our experiment, we have set the upper bound of the breathing rate which is $0.53 \mathrm{~Hz}$ (32 breath / min) with no modulation. The lower limit is estimated in our subjects able to breathe at rates of $0.4 \mathrm{~Hz}$ (24 breath / $\mathrm{min}$ ) in most individuals. So, if we analyze the FFT of the IC's, we will get high amplitude or peak in the region between $0.4 \mathrm{~Hz}$ to $0.52 \mathrm{~Hz}$. From our analysis we analyze the IC's by using FFT and

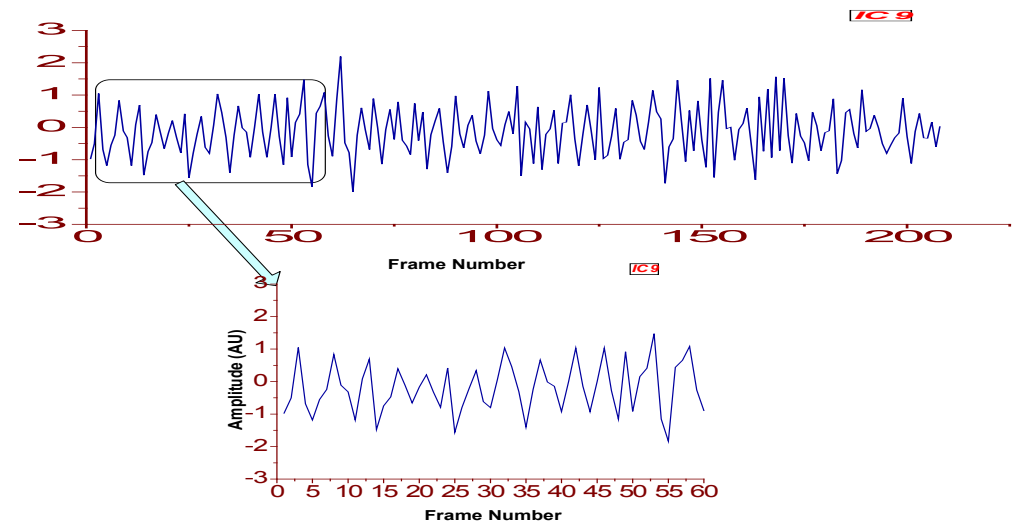

(a)

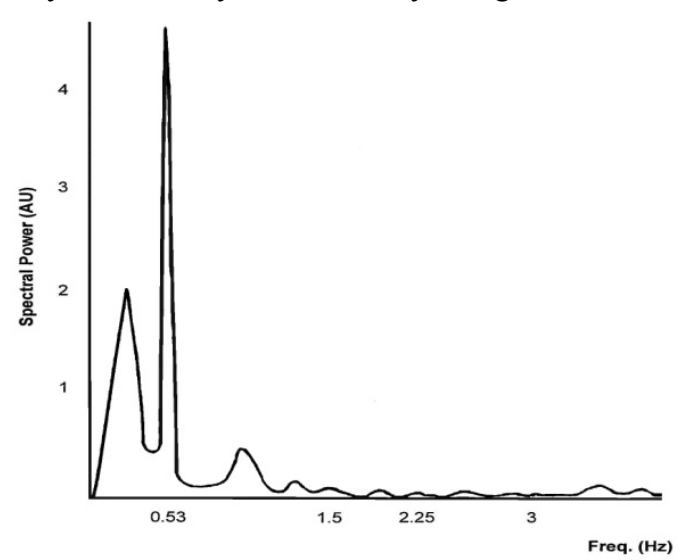

(b)

Fig. 8: Selected IC_9 with its magnified image (a) and its FFT (b). 


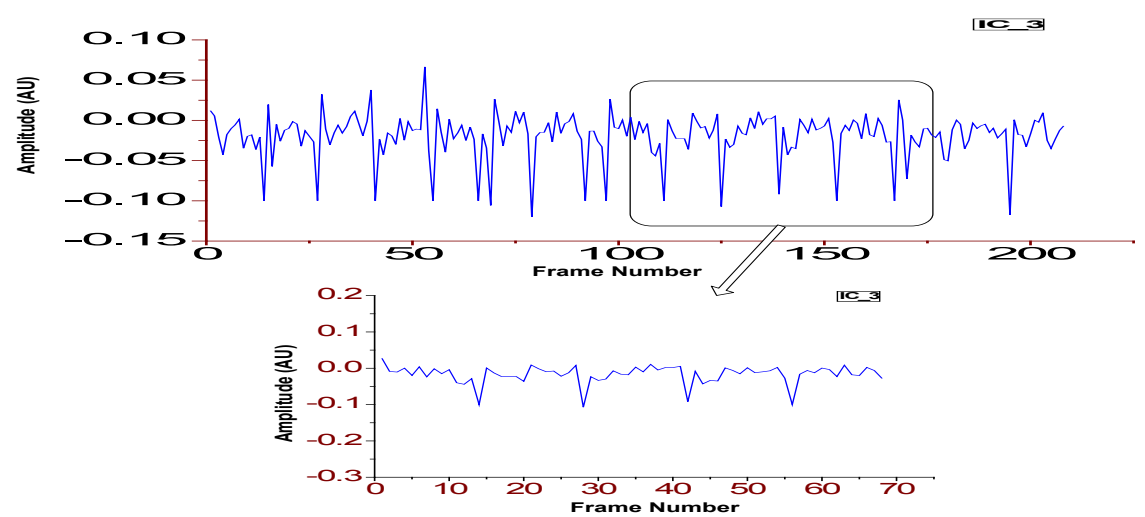

(a)

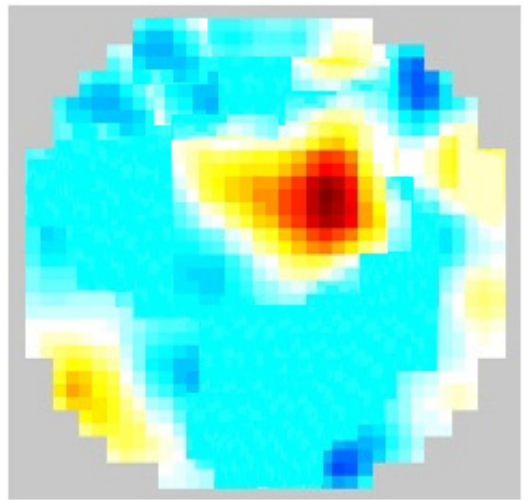

(b)

Fig. 9: Selected IC_3 with its magnified image and it's reconstructed Image

observe the high amplitude of the signals. The high spectrum sustained on the above region is selected as candidate IC for our experiment. By analysis, we have selected IC_2, IC_6, IC_7 and IC_9 as our desired signal. Then we generate the reconstruction image of the IC's and select the proper one for our desired respiration template RT. In Fig. 7 we have shown the reconstructed images of the selected IC's. From the reconstruction images and from the rigorous survey we came to the conclusion that IC 9 represent the best respiration signal and we have chosen this independent respiration signal as RT and go further for the next step of the experiment. In Fig. 8 we have shown the magnified image of the RT and its FFT.

For the next step we again use the preprocessing steps and the ICA algorithm as described in Fig. 5. After the first approximation and getting the RT, now we can get the remaining signal and get the cardiac signal as our desired template. By applying ICA on the remaining signal we can get the IC's again. From the IC's, we choose the right one as our desired template function $\mathrm{CT}$. Then we generate the reconstruction image of the IC's and select the proper one for our desired respiration template RT. In Fig. 9 we have shown the reconstructed images of the selected IC's (right column). Also, the remaining IC's which are selected and used for template function as cardiac template are shown in Fig. 10 .

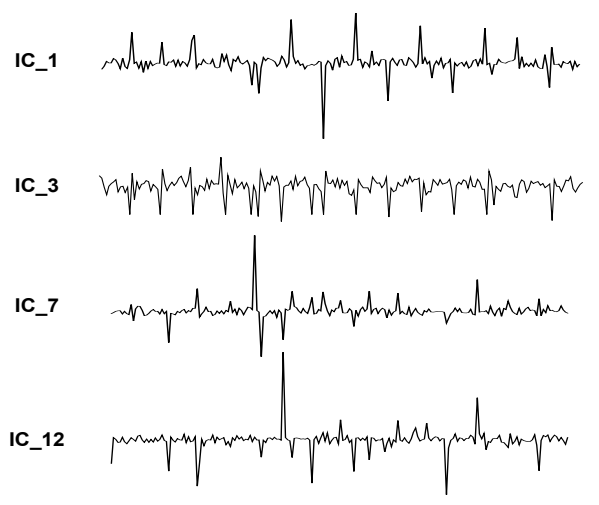

Fig. 10: Selected IC's from observed cardiac signals.

\section{Conclusion}

A new method for a dynamic extraction of cardiac and respiration changes in EIT image is proposed based on Independent Component Analysis. It does not rely on averaging over a number of heart cycles or on frequency domain filtering which is barely possible to implement properly. This method is reliable and further studies are going on to apply this method for different types of patients Also the processing speed of this method is so fast that a continuous real-time monitoring of patients seems to be possible in the near future using this method.

\section{Acknowledgement}

Special thanks to Professor Eung Je Woo of Kyung Hee University for providing me the environment to execute my experiments.

\section{References}

1. Barber DC, Seagar AD. Fast reconstruction of resistance images Clin. Phys. Physiol. Meas. 1987;8:47-54. http://dx.doi.org/10.1088/0143-0815/8/4A/006

2. Brown BH, Seagar AD. The Sheffield data collection system Clincal Phys. Physiol. Meas. 1987;8:91-97. http://dx.doi.org/10.1088/0143-0815/8/4A/012

3. Meier T, Luepschen H, Karsten J, Leibecke T, Großherr M, Gehring H, Leonhardt S, Assessment of regional lung recruitment and derecruitment during a peep trial based on electrical impedance tomography. Intensive Care Med. 2008; 34:543-50. http://dx.doi.org/10.1007/s00134-007-0786-9

4. Frerichs I, Dudykevych T, Hinz J, Bodenstein M, Hahn G, Hellige G. Funktionelle elektrische Impedanztomographie Intensivmed. Notfallmed. 2001; 91:39-50

5. Frerichs I, Hinz J, Herrmann P, Weisser G, Hahn G, Dudykevych T, Quintel M, Hellige G. Detection of local lung air content by electrical impedance tomography compared with electron beam CT . J. Appl. Physiol. 2002; 93:660-6. 
6. Frerichs I, Hinz J, Herrmann P, Weisser G, Hahn G, Quintel $\mathrm{M}$, Hellige G. Regional lung perfusion as determined by electrical impedance tomography in comparison with electron beam ct imaging. IEEE Trans. Med. Imaging. 2002; $21: 646-$ 52. http://dx.doi.org/10.1109/TMI.2002.800585

7. Luepschen H, Meier T, Grossherr M, Leibecke T, Karsten J, Leonhardt S. Protective ventilation using electrical impedance tomography. Physiol. Meas. 2007; 29:S247-60. http://dx.doi.org/10.1088/0967-3334/28/7/S18

8. Kunst PWA, Vonk-Noordegraaf A, Hoekstra OS, Postmus PE, de Vries PMJM. Ventilation and perfusion imaging by electrical impedance tomography: a comparison with radionuclide scanning. Physiol. Meas. 1998; 19:481-90. http://dx.doi.org/10.1088/0967-3334/19/4/003

9. Yan P, Mo Y. Using Independent Component Analysis for Electrical Impedance Tomography. SPIE-IS\&T. 2004; 5298: 448-454.

10. McArdle FJ, Suggett AJ, Brown BH, Barber DC. An assessment of dynamic images by applied potential tomography for monitoring pulmonary perfusion. Clin. Phys. Physiol. Meas. 1988; 9: 87-91.

http://dx.doi.org/10.1088/0143-0815/9/4A/015

11. Eyuboglu BM, Brown BH, Barber DC. In vivo imaging of cardiac related impedance changes. IEEE Eng. Med. Biol. Mag. 1989; 8: 39-45. http://dx.doi.org/10.1109/51.32404

12. Smit HJ, Vonk-Noordegraaf A, Boonstra A, de Vries PMJM, Postmus PE. Assessment of the pulmonary volume pulse in idiopathic pulmonary arterial hypertension by means of electrical impedance tomography. Respir. 2006; 73: 597-602. http://dx.doi.org/10.1159/000088694

13. Smit HJ, Handoko ML, Vonk Noordegraaf A, Faes TJC, Postmus PE, de Vries PMJM, Boonstra A. Electrical impedance tomography to measure pulmonary perfusion: is the reproducibility high enough for clinical practice? Physiol. Meas. 2003; 24: 491-499. http://dx.doi.org/10.1088/0967-3334/24/2/359

14. Deibele JM, Luepschen H, Leonhardt S. Dynamic separation of pulmonary and cardiac changes in electrical impedance tomography. Physiol. Meas. 2008; 29: S1-14. http://dx.doi.org/10.1088/0967-3334/29/6/S01
15. Grant CA, Pham T, Hough J, Riedel T, Stocker C, Schibler A. Measurement of ventilation and cardiac related impedance changes with electrical impedance tomography. Crit. Care. 2011; 15:R37. http://dx.doi.org/10.1186/cc9985

16. Leathard AD, Brown BH, Campbell J, Zhang F, Moricea H, Tayler D. A comparison of ventilatory and cardiac related changes in EIT images of normal human lungs and of lungs with pulmonary emboli. Physiol. Meas. 1994; 15: A137-146. http://dx.doi.org/10.1088/0967-3334/15/2A/018

17. Frerichs I, Pulletz S, Elke G, Reifferscheid F, Schadler D, Scholz J, Weiler N. Assessment of changes in distribution of lung perfusion by electrical impedance tomography. Respir. 2009; 77:282-91. http://dx.doi.org/10.1159/000193994

18. Pikkemaat R, Leonhardt S. Separation of ventilation and perfusion related signals within EIT-data streams. J. Phys. Conf. Ser. 2010; 224:012-028.

19. Zhukov L, Weinstein D, Johnson C. Independent component analysis for EEG source localization. IEEE Eng Med Biol Mag. 2000; 19(3): 87-96. http://dx.doi.org/10.1109/51.844386

20. Zhukov L, Weinstein D, Johnson CR. Independent Component Analysis for EEG Source Localization in Realistic Head Models. IEEE Engineering in Medicine and Biology. 2000; 3(19): 87-96. http://dx.doi.org/10.1109/51.844386

21. Arinen AH, Oja E. A Fast Fixed-Point Algorithm for Independent Component Analysis. Neural Computation, 1997; 9(7):1483-1492. http://dx.doi.org/10.1162/neco.1997.9.7.1483

22. Naik GR, Kumar DK. An Overview of Independent Component Analysis and Its Applications. Informatica, 2011; 35: 63-81.

23. Nguyen DT, Jin C, Thiagalingam A, McEwan AL. A review on electrical impedance tomography for pulmonary perfusion imaging. Physiol. Meas. 2012; 33: 695-706. http://dx.doi.org/10.1088/0967-3334/33/5/695 